\title{
Is the Deep Inspiration Breath-Hold Technique Superior to the Free Breathing Technique in Cardiac and Lung Sparing while Treating both Left-Sided Post-Mastectomy Chest Wall and Supraclavicular Regions?
}

\author{
Anupama Darapu $^{a} \quad$ Rajesh Balakrishnan ${ }^{a} \quad$ Patricia Sebastian $^{\text {a }}$ \\ Mohamathu Rafic Kather Hussain ${ }^{b}$ Paul Ravindran ${ }^{b}$ Subhashini John ${ }^{a}$ \\ ${ }^{a}$ Department of Radiotherapy, Christian Medical College, Vellore, India; ${ }^{b}$ Medical Physics, \\ Christian Medical College, Vellore, India
}

\section{Keywords}

Deep inspiration breath-hold · Post-mastectomy radiotherapy · Cardiac sparing · Free breathing
Abstract

Aims: To evaluate the efficacy of the deep inspirational breath-hold (DIBH) technique and its dosimetric advantages over the free breathing (FB) technique in cardiac (heart and left anterior descending artery [LAD]) and ipsilateral lung sparing in left-sided post-mastectomy fieldin-field conformal radiotherapy. DIBH is highly reproducible, and this study aims to find out its dosimetric benefits over FB. Materials and Methods: Nineteen left-sided mastectomy patients were immobilized using breast boards with both arms positioned above the head. All patients had 2 sets of planning CT images (one in FB and another in DIBH) with a Bio- 


\section{Case Reports in Oncology}

Case Rep Oncol 2017;10:37-51

DOI: $10.1159 / 000453607$

(c)

2017 The Author(s). Published by S. Karger AG, Basel www.karger.com/cro

Darapu et al: Is the Deep Inspiration Breath-Hold Technique Superior to the Free Breathing Technique in Cardiac and Lung Sparing while Treating both Left-Sided PostMastectomy Chest Wall and Supraclavicular Regions?

graph TruePoint HD CT scanner in the same setup. DIBH was performed by tracking the respiratory cycles using a Varian Real-Time Position Management system. The target (chest wall and supraclavicular region), organs at risk (OARs; ipsilateral lung, contralateral lung, heart, $L A D$, and contralateral breast), and other organs of interests were delineated as per the RTOG (Radiation Therapy Oncology Group) contouring guidelines. The single-isocenter conformal fields in the field treatment plans were generated with the Eclipse Treatment Planning System (Varian Medical Systems) for both FB and DIBH images, and the doses to the target and OARs were compared. The standard fractionation regimen of $50 \mathrm{~Gy}$ in 25 fractions over a period of 5 weeks was used for all patients in this study. Results and Discussion: The target coverage parameters (V95, V105, V107, and $D_{\text {mean }}$ ) were found to be $97.8 \pm 0.9,6.1 \pm 3.4,0.2$ \pm 0.3 , and $101.9 \pm 0.5 \%$ in the FB plans and $98.1 \pm 0.8,6.1 \pm 3.2,0.2 \pm 0.3$, and $101.9 \pm 0.4 \%$ in the DIBH plans, respectively. The plan quality indices (conformity index and homogeneity index) also showed $1.3 \pm 0.2$ and 0.1 for the FB plans and $1.2 \pm 0.3$ and 0.1 for the DIBH plans, respectively. There was a significant reduction in dose to the heart in the DIBH plans compared to the FB plans, with $p$ values of nearly 0 for the $\mathrm{V} 5, \mathrm{~V} 10, \mathrm{~V} 25, \mathrm{~V} 30$, and $\mathrm{D}_{\text {mean }}$ dosimetric parameters. The difference in ipsilateral lung doses between FB and DIBH showed statistically significant $p$ values, and the differences in mean doses were found to be 7, 15.7, 11.8 , and $10.7 \%$ for $\mathrm{V} 5, \mathrm{~V} 20, \mathrm{~V} 30$, and $\mathrm{D}_{\text {mean, }}$ respectively. There was a significant reduction in dose to the LAD in the DIBH compared to the FB plans. Conclusions: DIBH resulted in significant reductions in doses to the heart, LAD, and lungs, since with this technique there was an increase in the distance between the target and the OARs. With appropriate patient selection and adequate training, the DIBH technique is acceptable and achievable for radiotherapy to the chest, and therefore should be considered for all suitable patients, as this could result in fewer radiotherapy-related complications. However, this technique is time-consuming, since the setup is complex, results in an increased time for treatment delivery, and needs patient cooperation and technical expertise.

(C) 2017 The Author(s)

Published by S. Karger AG, Basel

\section{Introduction}

Breast cancer is the most common cancer to affect women in the world. Breast cancer is a complex disease which has many clinical, molecular, genetic, and morphological features. Estrogen, progesterone, and Her2/neu receptor status play an important role in determining the optimal personalized treatment for each patient. Breast cancer is typically treated with a combination of surgery, chemotherapy, hormone therapy, biological therapy, and radiation therapy based on the stage, hormonal status, and molecular and the genetic nature of the disease. Radiation is usually delivered using a linear accelerator. All patients who undergo breast conservation surgery and those who have undergone modified radical mastectomy with risk factors such as T3 lesions and above, node-positive disease, require radiotherapy. The meta-analysis by the Early Breast Cancer Trialists' Collaborative Group showed that the chances of local recurrence in women who received radiation therapy was $7 \%$, compared to $26 \%$ in those who did not receive radiotherapy [1]. The primary aim in breast cancer radiotherapy is to deliver the prescribed radiation dose (approx. $50 \mathrm{~Gy}$ ) to the entire breast - or, following mastectomy, to the entire chest wall - and to the regional nodes as indicated. This 


\section{Case Reports in Oncology}

is usually achieved by 2 beams that are tangentially oriented to the chest wall and opposite each other. The secondary aim is to avoid irradiating - or to reduce the dose - to the surrounding healthy tissues. However, this cannot always be achieved when organs are in close proximity to the treatment site. For left-sided breast cancer patients, the heart, major coronary arteries, and left lung tend to be coirradiated due to their proximity to the anterior chest wall. Therefore, radiotherapy can result in several complications, and many epidemiological studies have shown cardiac morbidity and mortality as major complications of leftsided breast/chest wall irradiation [2].

There is evidence from a retrospective study from Norway that the relative risk for ischemic heart disease is increased by $7.4 \%$ for every 1 -Gy increase in mean heart dose. This study was done in the older era, when patients were treated without CT planning or conformal techniques; it highlighted that any reduction in heart exposure to radiation is beneficial to the patient [3].

The results of 2 large trials (EORTC 22922 and MA 20) [4] demonstrated a benefit of locoregional radiation therapy, including treatment of the internal mammary nodes for nodepositive breast cancer. These results might result in an increased use of the internal mammary chain, the inclusion of which has been shown in various studies to increase the dose to the heart. This would necessitate the use of treatment techniques that minimize the dose to the heart, such as the deep inspiration breath-hold (DIBH) technique.

The aim of this study was to evaluate the efficacy of and patient compliance with the $\mathrm{DIBH}$ technique as well as its dosimetric advantages over free breathing (FB) techniques in cardiac and lung sparing among patients receiving radiotherapy to the left side of the chest wall.

\section{Methods}

This study was approved by the institutional review board. All patients were included in the study after they had signed the informed consent form for taking part in the study. Baseline echocardiography and pulmonary function tests were done. All the patients were informed well about the DIBH technique; they were trained in it and instructed to practice it for a period of at least 3 days before undergoing the planning CT scan. This study was only dosimetric, and no patient was treated with the DIBH technique. See Figure 1 for the study schema.

\section{Inclusion Criteria}

(1) Women with left-sided breast cancer requiring radiotherapy to the chest wall and supraclavicular region

(2) Patients who consented to undergo CT scanning in DIBH and in FB in the same setting and who permitted use of the images for research 


\section{Case Reports in Oncology}

Case Rep Oncol 2017;10:37-51

DOI: $10.1159 / 000453607$

(C) 2017 The Author(s). Published by S. Karger AG, Basel www.karger.com/cro

Darapu et al: Is the Deep Inspiration Breath-Hold Technique Superior to the Free Breathing Technique in Cardiac and Lung Sparing while Treating both Left-Sided PostMastectomy Chest Wall and Supraclavicular Regions?

Exclusion Criteria

(1) Patients who were incapable of holding their breath for any reason

(2) Patients who had primary or secondary flap reconstruction

Calibration of the Tracking System

Calibration of the infrared (IR) camera system (Varian Medical Systems, USA) was performed prior to acquisition of the images. Figure 2 shows the 6-neon marker localizer box, the video-assisted goggles, and the calibration setup of the IR camera. Initially, the localizer box was kept on the calibration test tool at the isocenter position using lasers. This was followed by a 10-step calibration procedure using Varian RPM software by placing the localizer box at various positions on the test tool with a fixed vertical table position (ideally at 200) and 3 different longitudinal positions to account for the coordinates of the various table positions during CT scanning. For each patient, this calibration check was performed to verify the shift in the $x, y$, and $z$ coordinates of the table (calibration validity) measured by the IR camera system. In this study, a maximum deviation of $\pm 3 \mathrm{~mm}$ from the calibration coordinates was accepted.

\section{Image Acquisition}

The planning CT images of the patients were acquired using a Biograph TruePoint HD CT scanner (Siemens, Germany). The patients were immobilized on a breast board with both arms abducted above the head. The planning CT images were acquired with a 3-mm slice thickness from the level of the 2 nd cervical vertebra to the adrenals with a field of view of $700 \mathrm{~mm}$ and the standard thorax imaging parameters. For all patients, 2 sets of planning CT images were acquired in the same setup, one during the normal breathing cycle (FB) and the other one during DIBH.

\section{Tracking of Respiratory Cycles}

After acquiring images of a patient during FB, the respiratory cycle of the patient was tracked by placing the localizer box on the patient in the treatment setup position. Initially, the baseline respiratory cycle was recorded. Following this, the patient was instructed through an audio system to hold the breath in deep inspiration for 15-20 s to record the respiratory phase in DIBH. Planning CT images were acquired after 2-3 successive trials done to verify the patient's ability to perform DIBH. The same acquisition parameter was used while acquiring the DIBH images.

\section{Treatment Planning}

The planning CT images were transferred to the Eclipse Treatment Planning System (Varian Medical Systems, USA). The FB and DIBH CT image sets were named as "CT FB" and "CT DIBH," respectively. The target (chest wall and supraclavicular region), the organs at risk (OARs; ipsilateral lung, contralateral lung, heart, left anterior descending artery [LAD], and contralateral breast) as per the RTOG contouring guidelines, and other organs such as the esophagus, liver, thyroid, and spine were delineated. Single-isocenter 3D conformal field-infield (FiF) treatment plans were generated for both FB and DIBH image sets. Two tangential- 


\section{Case Reports in Oncology}

ly oriented conformal beams and 1 direct anterior beam to the chest wall and supraclavicular region were used, and FiFs were created in the tangential fields. The standard fractionation regimen of 50 Gy in 25 fractions over a period of 5 weeks was used for all patients in this study. Plan evaluation was done for the parameters as shown in Table 1.

\section{Sample Size Calculation}

Based on the data reported by Bruzzaniti et al. [5], the mean (range) of the dose received by the lung in DIBH was 4.64 (3.32-6.11) units, with an SD of nearly 0.75 units [5]. The respective value with the FB method was 5.51 (3.54-8.84) units, with an SD of nearly 1.25 units. In order to show the difference of nearly 0.9 units that was statistically significant (with $\alpha$ and $\beta$ errors at 5 and 20\%, respectively), the sample size needed was nearly 19 subjects (who use both methods).

\section{Statistical Analysis}

To compare the dosimetric parameters of the 2 samples (FB and DIBH) which can be paired with one another, the population mean (true mean of the entire data) was used. Since our study involved comparison of 2 different planning techniques and the same sets of measurements in the same subjects, a statistical analysis by paired $t$ test was performed (e.g., target coverage, lung dose, heart dose, and LAD doses as determined from the FB and DIBH plans with same treatment technique were compared).

\section{Results}

All patients in our cohort were able to undergo the training required for using the DIBH technique. The patients' mean age in our cohort was 50 years (range 33-72). The mean body mass index of the cohort was 26 (range 17.3-34.2).

\section{Lung Volumes}

The primary aim of DIBH is to increase the volume of the lung and to move the heart away from the chest wall. In our study, we evaluated the difference in lung volumes in 3 age groups and between individual patients. The absolute volumes of the lung (combined and ipsilateral) measured for all patients are listed in Table 2. There was a significant difference in the absolute volume of the combined and ipsilateral lung obtained with FB and DIBH.

\section{Distance from the Heart to the Chest Wall}

The mean distance between the heart and the chest wall was analyzed on 2 different planes, one at the center of the heart in FB (taken as the reference plane in this study) to the center of the heart in DIBH and the other in the DIBH image corresponding to the center of the heart in FB. Figure 3 shows the location of the heart on the reference plane in FB and on the corresponding plane in DIBH.

The mean distances of the heart from the chest wall in the various age categories are shown in Figure 4. It was observed that the mean difference in the distance of the heart from the chest wall was considerable among the patients $<40$ years and $>55$ years, but there was no significant correlation observed between the age of a patient and the mean distance. 


\section{Case Reports in Oncology}

Case Rep Oncol 2017;10:37-51

DOI: $10.1159 / 000453607$

(C) 2017 The Author(s). Published by S. Karger AG, Basel www.karger.com/cro

Darapu et al.: Is the Deep Inspiration Breath-Hold Technique Superior to the Free Breathing Technique in Cardiac and Lung Sparing while Treating both Left-Sided PostMastectomy Chest Wall and Supraclavicular Regions?

\section{Dosimetric Analysis}

Various dosimetric parameters were used in our study to compare the treatment plans generated by FB and DIBH images of the patients. As target volumes, V95, V105, V107, and $D_{\text {mean }}$ were analyzed. Additionally, the plan quality indices, such as the conformity index (CI) and the homogeneity index (HI), were also compared. The dosimetric parameters were also compared for the OARs, which included the ipsilateral and contralateral lung, heart, LAD, and contralateral breast. The dosimetric parameters are summarized in Table 3.

\section{Target Volume Coverage}

The target coverage was comparable, and showed $97.8 \pm 0.9$ and $98.1 \pm 0.8 \%$ (for V95) for the FB FiF and the DIBH FiF plans, respectively. All the other parameters, including the CI and $\mathrm{HI}$, were also comparable between the plans. The $p$ values were found to be insignificant for all the parameters used.

\section{Ipsilateral Lung Doses}

The ipsilateral lung volumes were analyzed using 4 different dose volume parameters,

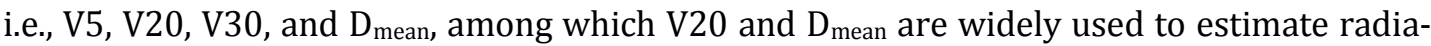
tion-induced side effects [6]. The 3 volume parameters V5, V20, and V30 were found to be better in the DIBH plans when compared to the FB plans, and they were statistically significant.

\section{Heart Doses}

The heart is the most critical OAR in patients receiving radiation therapy to the left chest wall. V25 and $D_{\text {mean }}$ are the most commonly used dose volume parameters for evaluating breast radiotherapy plans. V5 corresponds to the low-dose spread in the heart in percent. For better dosimetric evaluation, 2 additional dose volume parameters, V10 and V30, were used in our study. Table 3 shows the detailed report of the parameters analyzed for the heart in the FB and DIBH plans.

The statistical analysis showed that there was a significant reduction in dose to the heart in the DIBH plans for all the parameters that were analyzed as compared to the FB plans ( $p$ value of 0.0 for all the parameters). The tolerance limit of the heart is V25 $\leq 10 \%$, which means that $10 \%$ of the heart volume should not receive $>25$ Gy [7]. It was observed that V25 for the heart was $9.12 \pm 4.71 \%$ in the FB FiF plan, while it was $4.85 \pm 5.2 \%$ in the DIBH plan, which reduced the V25 by almost half with respect to the FB plan. Similar results were also found for V30, where the heart volume in FB showed $8.43 \pm 4.48 \%$, while in DIBH it showed $4.71 \pm 4.57 \%$. The other parameters, i.e., V5, V10, and $D_{\text {mean }}$, also showed statistically significant results ( $p$ value of nearly 0.0 ) for the heart volume, which clearly supports the DIBH technique for left-sided breast cancer patients to reduce radiation-induced side effects without compromising target coverage.

\section{LAD Doses}

The LAD is located in the anterior part of the heart, and this part of the heart is the one which receives the maximum dose while tangential fields are used to treat the chest wall. The doses received by the LAD are directly proportional to the risk of development of radiation-induced ischemic heart disease [8]. We evaluated LAD doses in the FB and DIBH plans 


\section{Case Reports in Oncology}

with 4 different dose volume parameters (V5, V10, V25, and $D_{\text {mean }}$ ). Since the volume of the LAD is only about $1 \mathrm{~cm}^{3}$, delineating and evaluating the dose to the LAD is a difficult task for the clinician. Delineation of the LAD in our study was restricted due to slice thickness, motion artifacts, and the washout of contrast before taking the second set of CT images (DIBH). Table 3 shows the LAD dose observed from the FB and DIBH plans. As very few studies in the literature dealt with doses to the LAD and no standard protocols for the estimation of LAD doses and limiting factors or tolerances have been defined, the parameters used for estimating doses to the heart were used for the LAD in our study. However, due to its location and considerable variation in its delineation according to the physicians' expertise, V5 for the LAD is unreliable and the SD observed in V5 is evidence for the same. In our study, a significant reduction of about $50 \%$ in doses to the LAD was observed in the DIBH plans in comparison with the FB plans. Furthermore, in the FB plans, there was lot of fluctuation in doses to the LAD due to respiratory motion, which could be avoided in the DIBH plans. The color wash of the $95 \%$ isodose at the level of the LAD in the same plane is illustrated in Figure 5. The dose-volume histograms of the target and all OARs in both plans are shown in Figure 6.

\section{Contralateral Lung, Breast, and Combined Lung Doses}

The dosimetric parameters were found to be similar, and there was no statistical significance regarding either of the 2 techniques, as the contralateral lung and breast are not part of the target volume in the tangential FiF technique used for treatment. The dosimetric parameters are summarized in Table 3.

\section{Discussion}

The advent of a combined-modality treatment approach in the management of breast cancer has led to improvement in local control and also in overall survival, which has resulted in longer survival and a stronger possibility of late complications [9]. Therefore, longterm morbidity and mortality with radiotherapy has become a concern, due to the fact that the doses received by OARs such as the heart and lungs result in higher chances of complications while delivering radiotherapy to the thoracic region - especially the left side [10]. Techniques of radiotherapy have improved over the years in an attempt to decrease doses to OARs and thereby reduce the probability of complications. The respiratory, cardiac, and gastrointestinal systems affect the movement of the target during radiotherapy, among which respiratory motion has a significant effect on intra- and interfractional treatment delivery. Hence, efforts have been made to account for and counteract the same, of which the DIBH technique is one of the most effective and reproducible methods and widely studied for breast cancer treatment $[7,11,12]$.

Our study was performed to evaluate the efficacy of and patient compliance with the DIBH technique and its dosimetric advantages over the FB technique in cardiac (heart and LAD) and ipsilateral lung sparing in left-sided post-mastectomy FiF conformal radiotherapy. The target volume coverage parameters in our study showed insignificant differences between the DIBH and FB plans. The quality indices CI and HI were also found to be similar. There was a significant reduction in dose to the heart in the DIBH plans compared to the FB 


\section{Case Reports in Oncology}

plans, with a $p$ value of 0.0 for the $\mathrm{V} 5, \mathrm{~V} 10, \mathrm{~V} 25, \mathrm{~V} 30$, and $\mathrm{D}_{\text {mean }}$ dosimetric parameters. There was a $46 \%$ reduction in dose to the heart for V25 as compared to the FB plan; in addition, the reduction in $\mathrm{D}_{\text {mean }}$ in DIBH as compared to FB was 2.05, and the $\mathrm{D}_{\text {mean }}$ in DIBH was 4.78 Gy, which was similar to that (5.3 Gy) seen in the study reported by Swamy et al. [13]. However, the data reported by Bruzzaniti et al. [5] showed a very low dose for $D_{\text {mean }}$ (1.2 Gy) for 8 patients. Nissen and Appelt [6] also reported a lower $\mathrm{D}_{\text {mean }}$ dose of $2.7 \mathrm{~Gy}$, but their study analyzed the doses for the DIBH and FB plans in 2 separate groups of patients. The dose reported for V30 Gy in the study by Swamy et al. [13] (3.3 $\pm 7.2 \%)$ was similar to our result $(4.7 \pm 4.6 \%)$.

The difference in mean doses between FB and DIBH was found to be $7,15.7,11.8$, and $10.7 \%$ for $\mathrm{V} 5, \mathrm{~V} 20, \mathrm{~V} 30$, and $\mathrm{D}_{\text {mean, }}$, respectively, and it was found to be statistically significant. These results were similar to the results reported by Swamy et al. [13] for ipsilateral lung doses.

In our study, there was a significant reduction in dose to the LAD in the DIBH plans compared to the FB plans, with a $p$ value of 0.0 for the V5, V10, V25, and $\mathrm{D}_{\text {mean }}$ dosimetric parameters. There was a $45.85 \%$ reduction in dose to the heart for V25 compared to the FB plan; in addition, the reduction in $\mathrm{D}_{\text {mean }}$ in DIBH as compared to FB was $8.18 \mathrm{~Gy}$, and the $\mathrm{D}_{\text {mean }}$ in DIBH was $9.66 \pm 2.6 \mathrm{~Gy}$, which was similar to that (5.3 $\pm 3 \mathrm{~Gy}$ ) seen in the study reported by Bruzzaniti et al. [5].

\section{Conclusion}

Use of the DIBH technique resulted in a significant reduction in doses to the heart, LAD, and lungs (OARs), since with this technique there was an increase in distance between the target and OARs. With DIBH, there was no compromise in doses to the target volume (planning target volume) with respect to coverage, with no underdosage or unacceptably high doses.

Radiotherapy to the chest wall with the DIBH technique therefore appears to be superior to radiotherapy with the FB technique. With appropriate patient selection and adequate training, the DIBH technique is acceptable and achievable for radiotherapy to the chest wall, and therefore should be considered for all suitable patients, as this could result in fewer radiotherapy-related complications. However, this technique is time-consuming, as the setup is complex, results in an increased time for treatment delivery, and needs patient cooperation and technical expertise.

\section{Acknowledgement}

This work was partly supported by a Fluid Research Grant, Christian Medical College, Vellore, India. 


\section{Case Reports in Oncology}

\section{Statement of Ethics}

This study was cleared by the Institutional Review Board and Ethics Committee of the hospital.

\section{Disclosure Statement}

None.

\section{Authors' Contributions}

Concept and design: all authors; administrative support: A.D., P.R., and S.J.; provision of study materials or patients: all authors; collection and assembly of data: all authors; data analysis and interpretation: all authors; manuscript writing: all authors; final approval of the manuscript: all authors.

\section{References}

1 Clarke M, Collins R, Darby S, Davies C, Elphinstone P, Evans V, Godwin J, Gray R, Hicks C, James S, MacKinnon E, McGale P, McHugh T, Peto R, Taylor C, Wang Y; Early Breast Cancer Trialists' Collaborative Group (EBCTCG): Effects of radiotherapy and of differences in the extent of surgery for early breast cancer on local recurrence and 15-year survival: an overview of the randomised trials. Lancet 2005;366:2087-2106.

-2 Bouillon K, Haddy N, Delaloge S, Garbay JR, Garsi JP, Brindel P, Mousannif A, Lê MG, Labbe M, Arriagada $\mathrm{R}$, Jougla E, et al: Long-term cardiovascular mortality after radiotherapy for breast cancer. J Am Coll Cardiol 2011;57:445-452.

3 Darby SC, Ewertz M, McGale P, Bennet AM, Blom-Goldman U, Brønnum D, Correa C, Cutter D, Gagliardi G, Gigante B, Jensen MB, et al: Risk of ischemic heart disease in women after radiotherapy for breast cancer. N Engl J Med 2013;368:987-998.

4 Whelan TJ, Olivotto IA, Parulekar WR, Ackerman I, Chua BH, Nabid A, Vallis KA, White JR, Rousseau P, Fortin A, et al: Regional nodal irradiation in early-stage breast cancer. N Engl J Med 2015;373:307-316.

5 Bruzzaniti V, Abate A, Pinnarò P, D’Andrea M, Infusino E, Landoni V, Soriani A, Giordano C, Ferraro AM, Strigari L: Dosimetric and clinical advantages of deep inspiration breath-hold (DIBH) during radiotherapy of breast cancer. J Exp Clin Cancer Res 2013;32:88.

6 Nissen HD, Appelt AL: Improved heart, lung and target dose with deep inspiration breath hold in a large clinical series of breast cancer patients. Radiother Oncol 2013;106:28-32.

7 Hanley J, Debois MM, Mah D, Mageras GS, Raben A, Rosenzweig K, Mychalczak B, Schwartz LH, Gloeggler PJ, Lutz W, Ling CC, et al: Deep inspiration breath-hold technique for lung tumors: the potential value of target immobilization and reduced lung density in dose escalation. Int J Radiat Oncol Biol Phys 1999;45:603-611.

8 El-Sherif O, Yu E, Xhaferllari I, Gaede S: Assessment of intrafraction breathing motion on left anterior descending artery dose during left-sided breast radiation therapy. Int J Radiat Oncol Biol Phys 2016;95:1075-1082.

-9 Cuzick J, Stewart H, Rutqvist L, Houghton J, Edwards R, Redmond C, Peto R, Baum M, Fisher B, Host H, et al: Cause-specific mortality in long-term survivors of breast cancer who participated in trials of radiotherapy. J Clin Oncol 1994;12:447-453.

10 Lee HY, Chang JS, Lee IJ, Park K, Kim YB, Suh CO, Kim JW, Keum KC: The deep inspiration breath hold technique using Abches reduces cardiac dose in patients undergoing left-sided breast irradiation. Radiat Oncol J 2013;31:239-246. 


\section{Case Reports in Oncology}

\begin{tabular}{l|l} 
DOI: $10.1159 / 000453607$ & C 2017 The Author(s). Published by S. Karger AG, Basel
\end{tabular} www.karger.com/cro

Darapu et al.: Is the Deep Inspiration Breath-Hold Technique Superior to the Free Breathing Technique in Cardiac and Lung Sparing while Treating both Left-Sided PostMastectomy Chest Wall and Supraclavicular Regions?

11 Mageras GS, Yorke E: Deep inspiration breath hold and respiratory gating strategies for reducing organ motion in radiation treatment. Semin Radiat Oncol 2004;14:65-75.

12 Mah D, Hanley J, Rosenzweig KE, Yorke E, Braban L, Ling CC, Leibel SA, Mageras G: Technical aspects of the deep inspiration breath-hold technique in the treatment of thoracic cancer. Int J Radiat Oncol Biol Phys 2000;48:1175-1185.

-13 Swamy ST, Radha CA, Kathirvel M, Arun G, Subramanian S: Feasibility study of deep inspiration breathhold based volumetric modulated arc therapy for locally advanced left sided breast cancer patients. Asian Pac J Cancer Prev 2014;15:9033-9038.

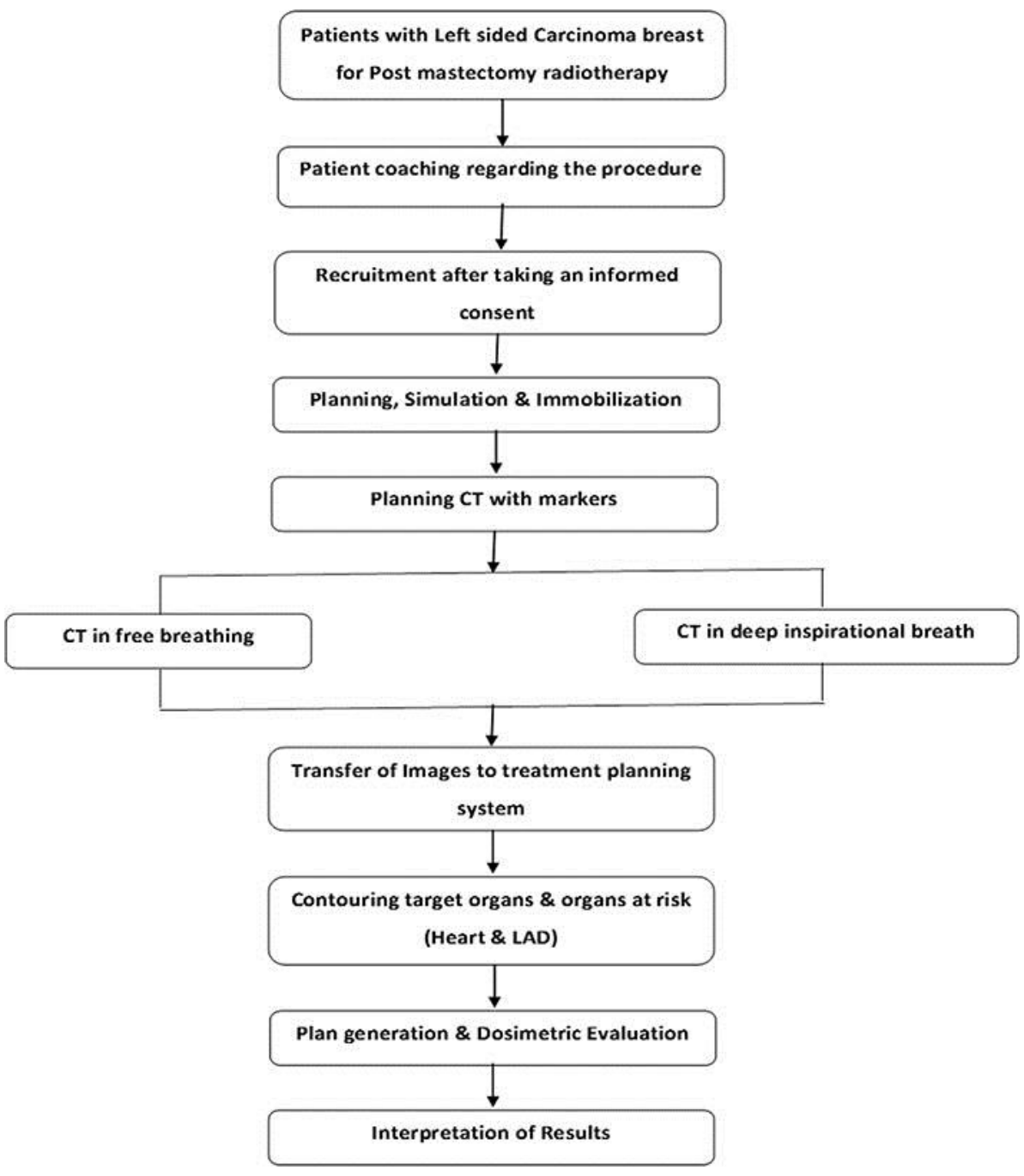

Fig. 1. Study schema. 


\section{Case Reports in Oncology}

Darapu et al.: Is the Deep Inspiration Breath-Hold Technique Superior to the Free Breathing Technique in Cardiac and Lung Sparing while Treating both Left-Sided PostMastectomy Chest Wall and Supraclavicular Regions?
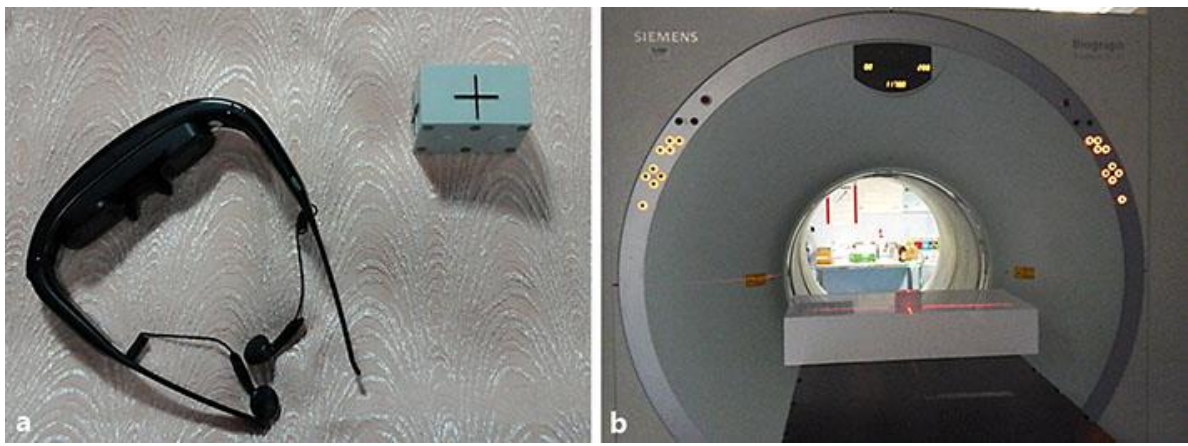

Fig. 2. a The 6-marker neon localizer box with crosshairs and goggle. b Calibration setup with fixed vertical couch position using lasers.

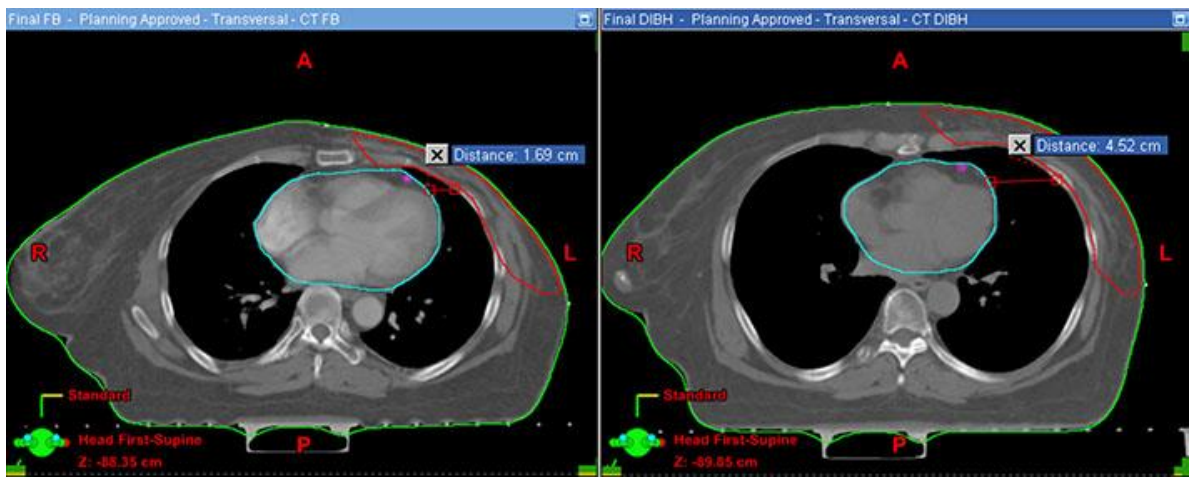

Fig. 3. Location of the heart on the reference plane in FB and on the corresponding plane in DIBH. 


\section{Case Reports in Oncology}

Darapu et al.: Is the Deep Inspiration Breath-Hold Technique Superior to the Free

Breathing Technique in Cardiac and Lung Sparing while Treating both Left-Sided Post-

Mastectomy Chest Wall and Supraclavicular Regions?

\section{Heart distance from chest wall}

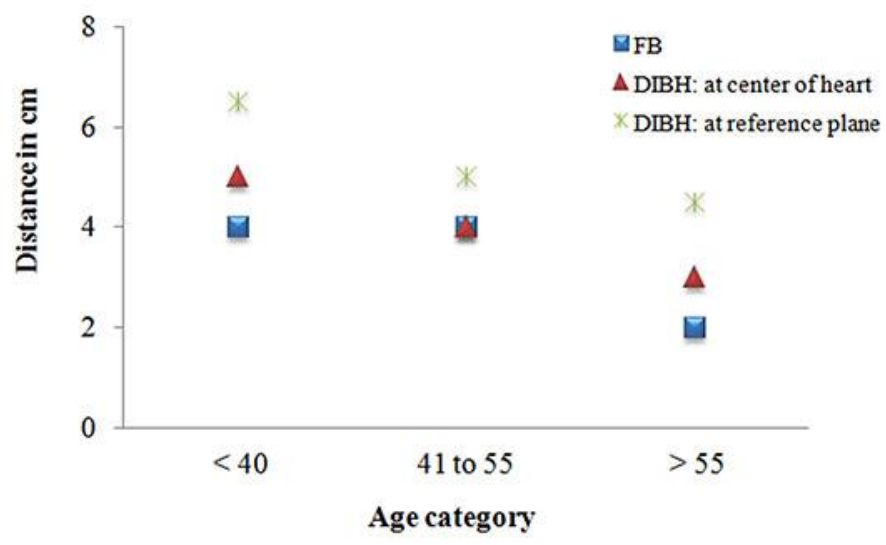

Fig. 4. Mean heart distance from chest wall in FB and DIBH (on 2 planes).

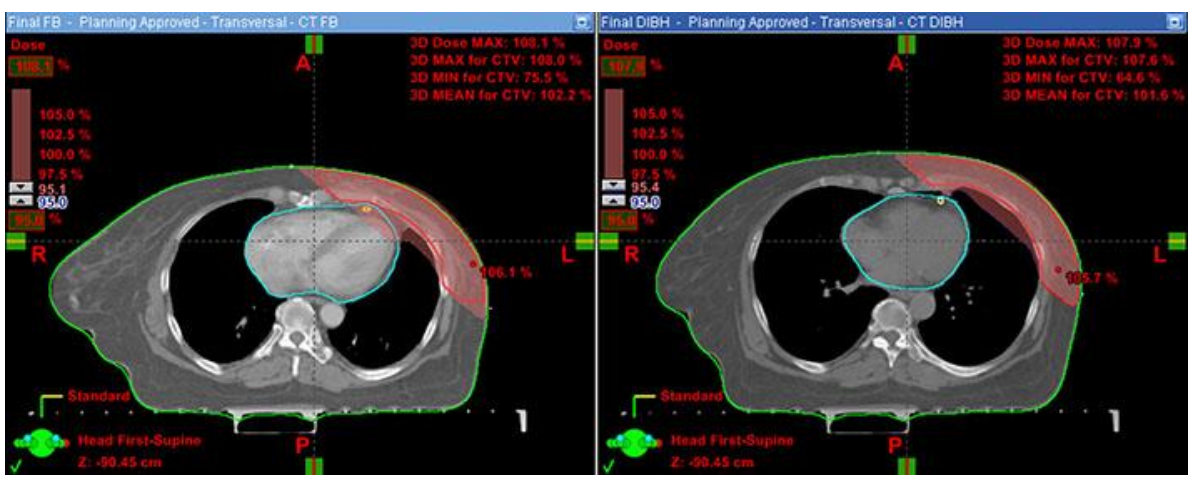

Fig. 5. Color wash of the $95 \%$ isodose at the level of the LAD. 


\section{Case Reports in Oncology}

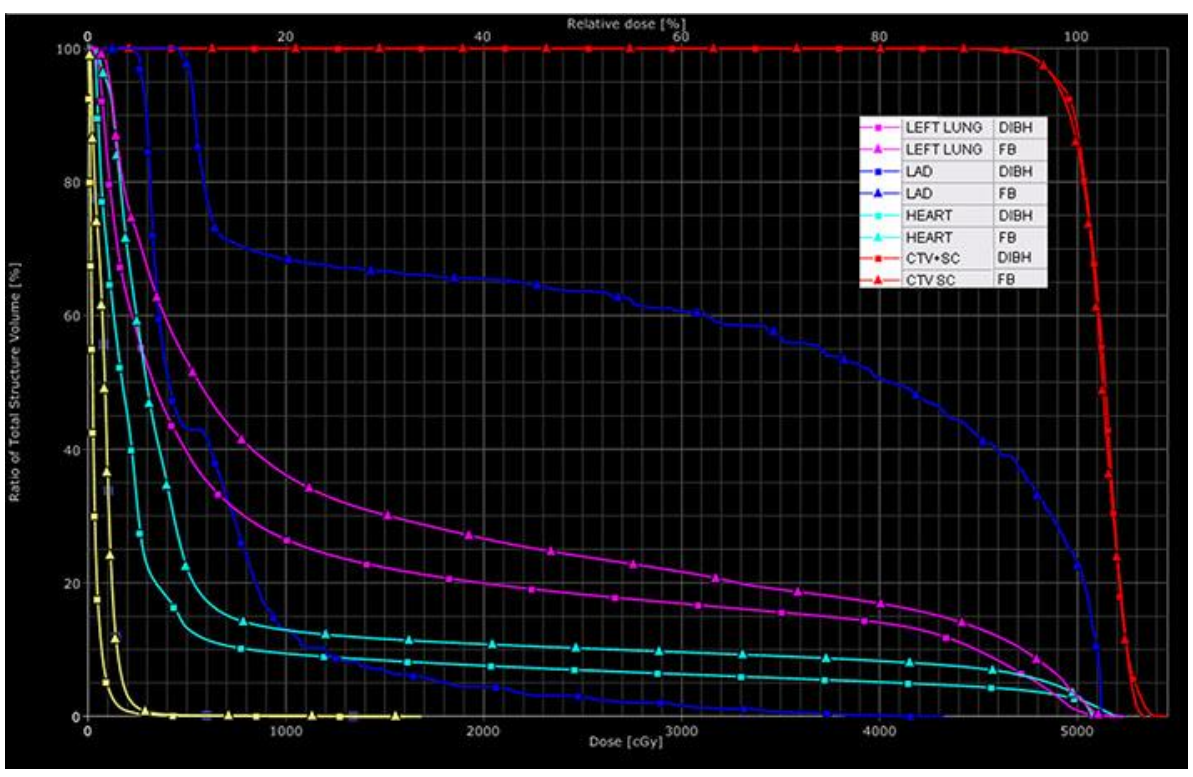

Fig. 6. Plan computed dose-volume histogram of FB (triangles) and DIBH (squares).

Table 1. Dosimetric parameters evaluated

\begin{tabular}{llll}
\hline $\begin{array}{l}\text { Subject } \\
\text { No. }\end{array}$ & Structures & Dosimetric parameters & $\begin{array}{l}\text { Cumulative } \\
\text { DVH mode }\end{array}$ \\
\hline 1 & Target (CTV plus SC) & $\begin{array}{l}\text { V95\%, V105\%, V107\%, D98\%, D2\%, Dmin, } \\
\mathrm{D}_{\text {max, }} \text { and Dmean (dose-volume coverage) }\end{array}$ & Relative \\
\hline 2 & Target (CTV plus SC) & HI and CI (plan quality parameters) $^{\text {a }}$ & Relative \\
\hline 3 & Ipsilateral lung & V5 Gy, V20 Gy, V30 Gy, and Dmean & Absolute \\
\hline 5 & Combined lung & V5 Gy, V20 Gy, V30 Gy, and Dmean & Absolute \\
\hline 6 & Contralateral lung & V5 Gy and Dmean & Absolute \\
\hline 7 & Heart & V5 Gy, V25 Gy, V30 Gy, and Dmean & Absolute \\
\hline 8 & LAD & V5 Gy, V10 Gy, V25 Gy, and Dmean & Absolute \\
\hline
\end{tabular}

DVH, dose-volume histogram; CTV, clinical target volume; SC, supraclavicular region; LAD, left anterior descending artery. ${ }^{a} \mathrm{HI}$ and CI calculated as per the ICRU report 83. 
Table 2. Absolute volumes of the lung in FB and DIBH $\left(\mathrm{cm}^{3}\right)$

\begin{tabular}{|c|c|c|c|c|c|c|}
\hline \multirow{2}{*}{$\begin{array}{l}\text { Patient } \\
\text { No. }\end{array}$} & \multicolumn{3}{|c|}{ Combined lung volume } & \multicolumn{3}{|c|}{ Left lung volume } \\
\hline & FB & DIBH & difference & FB & DIBH & difference \\
\hline 1 & $1,915.7$ & $3,644.4$ & $1,728.7$ & 974.2 & $1,867.3$ & 893.1 \\
\hline 2 & $1,803.1$ & $3,736.6$ & $1,933.6$ & 853.5 & $1,751.3$ & 897.8 \\
\hline 3 & $1,880.6$ & $4,057.9$ & $2,177.3$ & 903.0 & $1,910.3$ & $1,007.3$ \\
\hline 4 & $1,718.1$ & $3,392.8$ & $1,674.7$ & 774.3 & $1,637.1$ & 862.8 \\
\hline 5 & $2,642.0$ & $3,693.2$ & $1,051.2$ & $1,203.0$ & $1,912.0$ & 709.1 \\
\hline 6 & $2,047.6$ & $3,713.5$ & $1,665.9$ & $1,015.0$ & $1,859.3$ & 844.3 \\
\hline 7 & $2,175.4$ & $3,884.9$ & $1,709.5$ & 988.1 & $1,814.9$ & 826.8 \\
\hline 8 & $2,312.2$ & $3,700.5$ & $1,388.3$ & $1,004.4$ & $1,685.8$ & 681.4 \\
\hline 9 & $2,283.2$ & $3,246.2$ & 963.1 & $1,128.1$ & $1,584.6$ & 456.6 \\
\hline 10 & $2,718.3$ & $2,758.6$ & 40.2 & $1,146.8$ & 1,161.9 & 15.1 \\
\hline 11 & 1,707.8 & $3,020.0$ & 1,312.2 & 752.3 & 1,377.0 & 624.7 \\
\hline 12 & 1,797.3 & $3,714.0$ & 1,916.7 & 837.6 & $1,742.3$ & 904.7 \\
\hline 13 & 1,868.7 & $3,556.9$ & 1,688.2 & 835.3 & $1,695.8$ & 860.5 \\
\hline 14 & $2,166.8$ & $3,693.4$ & $1,526.7$ & 903.1 & $1,641.8$ & 738.7 \\
\hline 15 & $2,061.8$ & $3,261.6$ & 1,199.8 & 919.8 & $1,524.9$ & 605.1 \\
\hline 16 & 1,357.5 & $2,133.0$ & 775.5 & 516.3 & 835.8 & 319.4 \\
\hline 17 & $1,736.5$ & $3,252.9$ & $1,516.4$ & 808.2 & 1,518.6 & 710.4 \\
\hline 18 & $1,924.2$ & $3,950.3$ & $2,026.1$ & 910.3 & $1,844.6$ & 934.3 \\
\hline 19 & $1,725.5$ & $3,921.5$ & $2,196.0$ & 724.7 & $1,765.8$ & $1,041.2$ \\
\hline
\end{tabular}


Table 3. Dosimetric parameters compared between FB and DIBH plans

\begin{tabular}{|c|c|c|c|c|c|c|c|c|c|}
\hline \multirow[t]{2}{*}{ Parameter } & \multicolumn{4}{|l|}{$\mathrm{FB}, \%$} & \multicolumn{4}{|c|}{ DIBH, \% } & \multirow{2}{*}{$\begin{array}{l}p \\
\text { value }\end{array}$} \\
\hline & $\min$. & $\max$ & mean & SD & $\min$. & $\max$ & mean & SD & \\
\hline \multicolumn{10}{|c|}{ Target volume } \\
\hline V95 & 96.1 & 99.1 & 97.8 & 0.9 & 96.3 & 99.1 & 98.1 & 0.8 & 0.15 \\
\hline V105 & 0.4 & 11.8 & 6.1 & 3.4 & 0.2 & 11.1 & 6.1 & 3.2 & 1 \\
\hline V107 & 0 & 0.9 & 0.2 & 0.3 & 0 & 0.9 & 0.2 & 0.3 & 0.95 \\
\hline $\mathrm{D}_{\text {mean }}$ & 100.9 & 102.8 & 101.9 & 0.5 & 101.1 & 102.5 & 101.9 & 0.4 & 0.95 \\
\hline $\mathrm{CI}$ & 1 & 1.9 & 1.3 & 0.2 & 0.9 & 2.2 & 1.2 & 0.3 & 0.238 \\
\hline $\mathrm{HI}$ & 0.1 & 0 & 0.1 & 0 & 0.1 & 0 & 0.1 & 0 & 0.523 \\
\hline \multicolumn{10}{|c|}{ Ipsilateral lung dose } \\
\hline V5 & 38.01 & 56.73 & 48.82 & 6.17 & 34.99 & 54.2 & 45.18 & 5.69 & 0.015 \\
\hline V20 & 16.39 & 32.21 & 25.44 & 4.32 & 15.97 & 27.7 & 21.45 & 3.42 & 0.00 \\
\hline V30 & 11.02 & 25.7 & 20.09 & 3.75 & 12.4 & 22.3 & 17.72 & 3.07 & 0.003 \\
\hline $\mathrm{D}_{\text {mean }}$ & 9.42 & 16.42 & 13.59 & 2.05 & 9.07 & 15.3 & 12.13 & 1.73 & 0.00 \\
\hline \multicolumn{10}{|c|}{ Combined lung dose } \\
\hline V5 & 14.6 & 26.5 & 22.26 & 3.44 & 15.55 & 25.2 & 21.07 & 3.13 & 0.100 \\
\hline V20 & 6.27 & 15.5 & 11.62 & 2.36 & 6.6 & 12.6 & 10.73 & 1.78 & 0.340 \\
\hline V30 & 4.2 & 12.6 & 9.18 & 2.02 & 4.85 & 10.5 & 8.25 & 1.129 & 0.018 \\
\hline$D_{\text {mean }}$ & 4.1 & 8.19 & 6.49 & 1.12 & 4.38 & 7.2 & 5.91 & 0.908 & 0.011 \\
\hline \multicolumn{10}{|c|}{ Contralateral lung dose } \\
\hline V5 & 0 & 0.18 & 0.025 & 0.56 & 0 & 0.4 & 0.06 & 0.11 & 0.011 \\
\hline$D_{\text {mean }}$ & 0.3 & 1.01 & 0.517 & 0.212 & 0.28 & 0.84 & 0.495 & 0.16 & 0.645 \\
\hline \multicolumn{10}{|l|}{ Heart } \\
\hline V5 & 9.77 & 46.7 & 21.44 & 9.43 & 4.18 & 40.4 & 15.74 & 9.15 & 0.00 \\
\hline V10 & 4.43 & 24.15 & 12.42 & 5.65 & 0.5 & 23.3 & 7.72 & 5.98 & 0.00 \\
\hline V25 & 2.55 & 18.3 & 9.12 & 4.71 & 0 & 18.4 & 4.85 & 5.21 & 0.00 \\
\hline V30 & 2.22 & 17.2 & 8.43 & 4.48 & 0 & 17.4 & 4.71 & 4.57 & 0.00 \\
\hline $\mathrm{D}_{\text {mean }}$ & 3.1 & 12.35 & 6.827 & 2.69 & 1.81 & 12.09 & 4.775 & 2.59 & 0.00 \\
\hline \multicolumn{10}{|l|}{ LAD } \\
\hline V5 & 14.2 & 97.56 & 25.24 & 53.47 & 12.51 & 81.84 & 41.3 & 19.81 & 0.03 \\
\hline V10 & 0 & 86.5 & 39.52 & 24.61 & 0 & 50 & 19.94 & 15.43 & 0.002 \\
\hline V25 & 0 & 72.48 & 31.91 & 24.47 & 0 & 45 & 12.48 & 15.74 & 0.002 \\
\hline $\mathrm{D}_{\text {mean }}$ & 4 & 36.94 & 17.84 & 10.73 & 3.06 & 23.16 & 9.66 & 6.454 & 0.001 \\
\hline \multicolumn{10}{|c|}{ Contralateral breast } \\
\hline V5 & 0 & 4.56 & 0.44 & 1.038 & 0 & 6.61 & 0.75 & 1.698 & 0.16 \\
\hline$D_{\max }$ & 3.47 & 47.59 & 20.26 & 10.96 & 3.46 & 47.7 & 18.83 & 12.35 & 0.39 \\
\hline $\mathrm{D}_{\text {mean }}$ & 0.08 & 1.47 & 0.45 & 0.333 & 0.2 & 2.17 & 0.66 & 0.48 & 0.001 \\
\hline
\end{tabular}

